\title{
An ObJect Detection Navigator TO ASSIST THE VISUALLY IMPAIRED USING ARTIFICIAL INTELLIGENCE AND COMPUTER VISION
}

\author{
Ethan $\mathrm{Wu}^{1}$, Jonathan Sahagun ${ }^{2}$ and $\mathrm{Yu} \mathrm{Sun}{ }^{3}$ \\ ${ }^{1}$ St John Bosco High School, Bellflower, CA 90706 \\ ${ }^{2}$ California State University, Los Angeles, Los Angeles, CA, 90032 \\ ${ }^{3}$ California State Polytechnic University, Pomona, CA, 91768
}

\begin{abstract}
The advent and worldwide adoption of smartphones has enriched the lives of many people. However, one particular group--the visually impaired--still need specific apps to help them with their daily lives. Thus, I'm developing this Smart app to specifically help the visually-impaired. Specifically, I hope to integrate the functions of Google Maps into the Smart App. While Google Maps functions well as a GPS for the average person without any impairment, I'm adding additional features to the Smart app so that it would guide the eye-sight impaired. For example, I will use the camera of the Smartphone to guide the user such that it would take the user to the desired destination. Thus, using the inherent functions (camera) of a phone, the Smart app can gently and safely guide any sight-impaired person to a predetermined destination by walking. One can think of Smart app as an improvement upon Google Maps -- for the visually impaired.
\end{abstract}

\section{KEYWORDS}

Object detection, Google Maps, iOS, Android.

\section{INTRODUCTION}

The primary motivation to create this particular smartphone app is to help visually impaired persons to experience the modern convenience of Google Maps $[4,5]$ while enriching their lives. Toward this goal, I had previously designed a similar product 2 years ago called "Sonar Stick" [6] that acts as a "GPS guided" walking cane for the visually impaired. Thus, my motivation to help the visually-impaired goes back several years. I just believe that everyone should enjoy the modern convenience of technology, and that technology is meant to transform every one of us, and not leave anyone behind.

However, after I completed my design of the "Sonar Stick," I felt that mass-producing them would become challenging as well as time-consuming. Thus, I began to think of different ways to allow my idea to become fruition while bringing such a product to a wider audience. With the advent and the popularity of smartphones, it's natural that I'm now proceeding to develop an app, which will reach a wider audience without the obstacles of manufacturing. In other words, to realize my goal, I've changed course from hardware (Sonar Stick) to software (Sonar Object Detection) app. With this approach, I feel very confident that I'm able to help the visuallyimpaired and transform their lives in a much positive way. 
Currently, perhaps Google Maps is the closest tool to help navigate us to destinations we enter. However, Google Maps is intended for the mass, as it works extremely well for those who have regular eyesight. However, for the visually-impaired, Google Maps (even if its speaking function is activated) still have a drawback such that the visually-impaired person does not know WHERE to start. For example, Google maps would start off navigation by announcing go West on Main Street, and then turn left on Washington Ave...."

For the visually impaired, they wouldn't know which direction to start, and it's predictable that someone with bad eyesight could start off in the wrong direction (and possibly spin in circles). That's where my smartphone app comes in--to eliminate this very problem. Specifically, the "Sonar Object Detection" app uses the phone's camera to act as "eyes" for the visually-impaired, such that s/he would start off with the correct direction. Thus, my app would synchronize the very best features of Google Maps and the Smartphone (camera). Together, the app and Google Maps would guide the user to the destination safely--in a time that's comparable to ordinary people. The visually-impaired then would enjoy the benefits of modern technology like the rest of us.

So, one can view my smartphone app as a one to improve upon existing technology (Google Maps). For technology to improve, one possible way is to improve upon existing technology. That's the route I'm taking and I sincerely believe it would make a great difference in people's lives.

In this paper, we follow the same line of research and method by Google, especially Google Maps. Since this is an existing method and product, there's no need to "invent" another Google Maps. Instead, we hope to improve upon Google Maps such that the visually-impaired would enjoy the benefits of Google Maps. As outlined above, we are making this app to tailor specifically to the visually-impaired.

Specifically, we are designing this app such that the user could hold up a smartphone. A quick Google search reveals that there are currently no products or app that cater to the visuallyimpaired in the area of GPS guidance. Unfortunately, the visually-impaired have been ignored, and ignored for far too long. We seek to change this apathy for the blind and visually-impaired. Therefore, we believe our method will result in helping others, a very worthwhile goal.

To testify that our app actually works we decided to head to the streets and actually test our app out. First, we opened a beta version of the app on our phones and started walking down a variety of paths, from streets next to a busy road to streets next to a shopping plaza. Our app began to read out what was in front of us, showing that our app actually worked as we expected.

When walking on the street, mimicking what a visually-impaired person would do with a cane, we practiced scanning our phone from left to right, and the app announced out loud what the nearby objects were immediately and accurately. On a busy street, where the biggest danger to a visually impaired person would be cars, the app accurately announced incoming cars and traffic lights, going so far as to identify the shape of the car, such as a track. On the street next to a shopping plaza, the app properly identified large plants, trees, and also people as they walked by. Checking accuracy was key to this experiment to ensure that when this is hopefully used by the visually impaired, it will be better than the cane they typically use and will truly help them.

We knew that this experiment proving that our app's capabilities have no mistake was not enough, so we decided to contact a foundation called the American Foundation for the Blind to help us review our app and also to give us any suggestions on anything that we could improve to 
achieve our goals which is to ultimately allow visually impaired people to successfully use our app and help their daily life.

The rest of the paper is organized as follows: Section 2 gives the details on the challenges that I met during the experiment and designing the sample; Section 3 focuses on the details of our solutions corresponding to the challenges that I mentioned in Section 2; Section 4 presents the relevant details about the experiment I did, following by presenting the related work in Section 5 . Finally, Section 6 gives the conclusion remarks, as well as pointing out the future work of this project.

\section{Challenges}

\subsection{Challenge 1}

Initially, the biggest challenge I faced was coding a functional app that incorporated all of my ideas and goals. At the time, I had no experience in coding or creating an app, which was challenging because that meant I had no way of channeling my ideas into reality.

So, I looked for resources elsewhere. I reached out to Professor Jonathan Sahagun of Cal State Pomona, shared my ideas, and requested assistance. Professor Sahagun is a Computer Science professor, and he taught me how to code and how to create this app.

Learning to code was difficult since it was a brand-new language, and I found myself having trouble navigating a brand-new unfamiliar world. As a result, starting the creation of the app was the most difficult. Gradually, however, various aspects of coding started to make sense, and with some guidance from Professor Sahagun, I was eventually able to create the beta version of this app.

\subsection{Challenge 2}

When creating this app, I did so from an Android standpoint, both because Androids are a very commonly used phone and because I had one myself. So, the resulting app that I currently have is very accurate and useful on an Android [7, 8, 9]. However, this app has not yet been adapted for iOS [10] devices.

When attempting to adapt the app for iOS, I encountered multiple glitches and bugs. This is a critical challenge because iOS phones are extremely common amongst the visually impaired phone. This app was created to help the visually impaired live an easier and more free life, so for this app to only be restricted to Android users would be the opposite of our intention to aid the visually impaired.

At the moment, I have not yet figured out how to properly have this app be available on iOS. I have made multiple attempts and thus, multiple improvements, but it appears there's still some time needed before I can adapt the app so it is suitable for everyone. This is a challenge I must and will overcome for the sake of the visually impaired.

\subsection{Challenge 3}

It has been challenging to figure out how to spread the information about this new app. This app will be of great aid to the visually impaired population, yet I originally had no way of reaching 
out to them and letting them know of this app. I considered advertisements or social media presence, yet I realized those would not be the most efficient way to reach the visually impaired. To solve this challenge, I contacted the American Foundation for The Blind [11, 12, 13], asking on how best to distribute knowledge of the app to reach the visually impaired. This foundation offered a partnership for people who are aiding the visually impaired. Within this partnership, they would help us spread the information of this app to the visually impaired so they can use our app.

With this foundation, an institution with direct and wide access to the visually impaired, I know that our app will be able to reach more people.

\section{Solution}

The "Sonar Object Detector" App is a smartphone app that directs a visually-impaired person to walk to a destination using the smartphone camera along with Google Map Instructions. Once the user downloads this app (currently the app is only compatible with Android phones), the user can activate the app by tapping on their phone to begin use.

Once activated, the "Sonar Object Detector" app will ask the user to input an address, much like using Google Maps. Once the address is inputted, the app will synchronize with Google Maps and then activate the phone's camera. The user is then instructed to hold up the camera such that the camera can "see" the direction and, together with the app, locate the exact location of the user on Google Maps.

Thereafter, the app will gently guide the user to his/her destination, with the camera acting as "eyes" and the phone's speaking blaring out the audio directions of Google Maps.

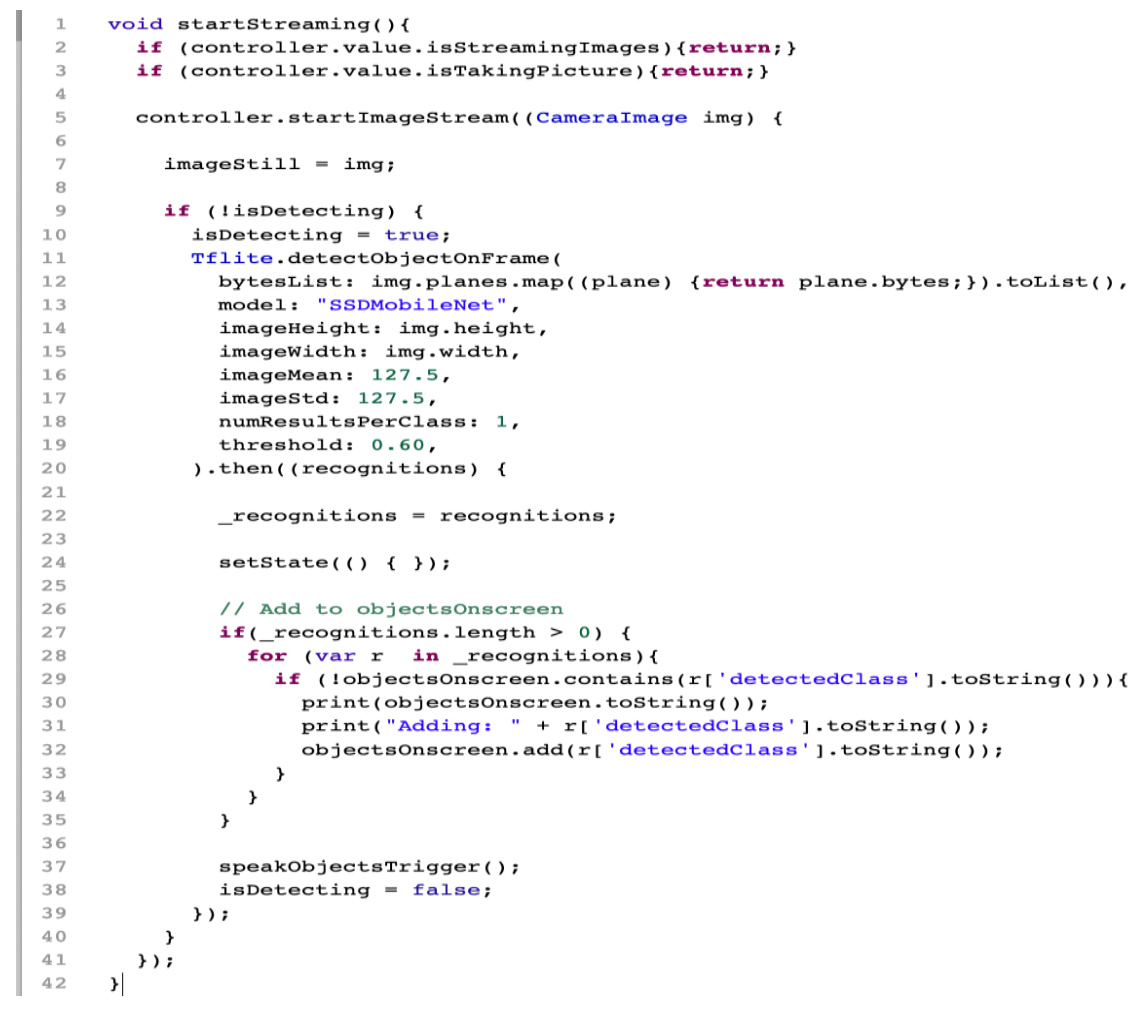

Figure 1: core code for starting the camera and detecting objects from the camera stream 
There are multiple components to this system. First is the camera, which the user moves from side to side in order to capture their surroundings. The camera acts as the "eyes" of the person, absorbing the objects around the user. Next is coding of the app, which allows the recording information from the camera to be transferred into actual objects that are spoken out. The next component is the speaker, which announces the type of objects/people to the user. And of course, the final component is this app's connection to Google Maps, which verbalizes the directions all while the "Sonar Object Detector" app describes the surrounding environment. This way, the visually-impaired user can reap the benefits of technology that the rest of us enjoy.

Above is the function used to start the camera and detect objects from the camera stream. The function starts by returning if the camera controller is already streaming. Line 4 of the function starts the camera stream. An anonymous function is created as the argument of the camera controller's startstream function. The anonymous function takes a frame from the camera stream. In the function a lock, isDetecting, is used to make sure tensorflow is only ever running once. The TensorFlowLite package is used for object detection. The camera image from the camera stream is passed to the tflite's objectDetectionFrame function with some other parameters such as requiring the object detection be $60 \%$ confident in its detections. When the detection has completed it returns a list of recognitions that contain the objects it has detected, their location within the camera image and their size. That list is used to draw rectangles on top of the camera feed in the app and to have the app speak the objects that were detected.

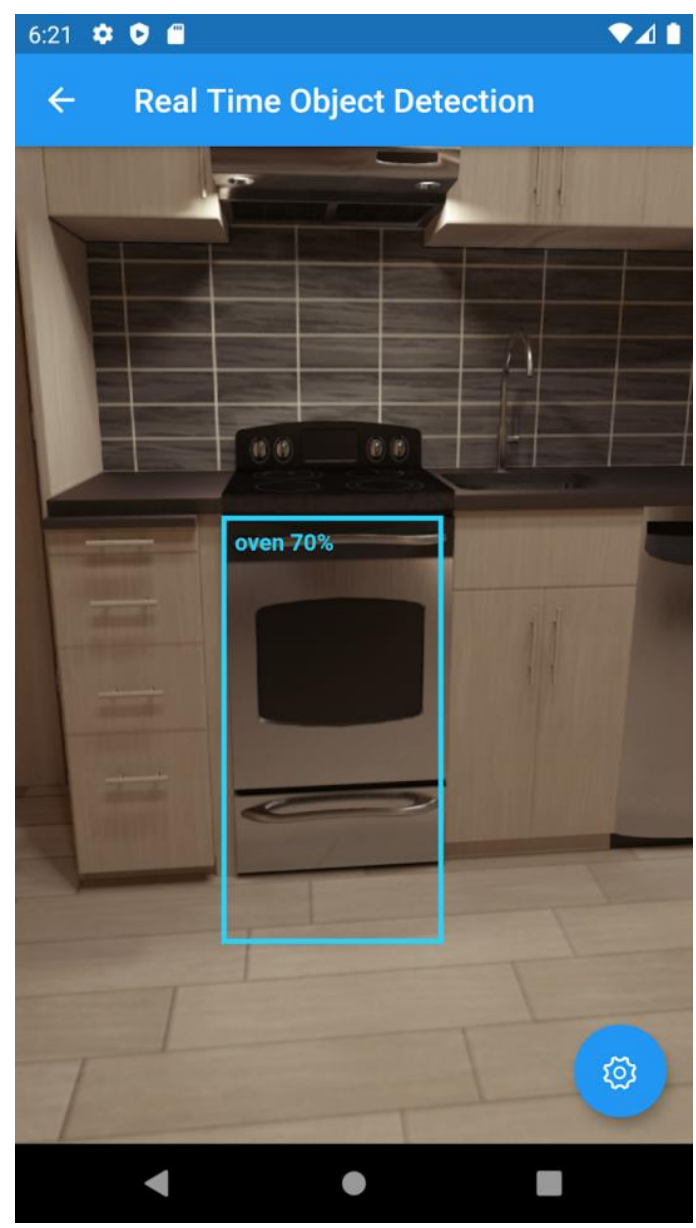

Figure 2: an example of object detection 


\section{EXPERIMENT}

In order to evaluate the accuracy of my app, I had to test it in as many types of situations a visually impaired person would experience in their everyday life. So, I divided my experimentation into two aspects - walking outdoors and indoors in a variety of situations.

I tested the beta version of my app along 3 different scenarios: a busy street full of cars, a shopping plaza, and a pedestrian-filled area. So, the independent variable was the type of area I was in while the dependent variable were the objects recorded. I recorded the types of objects that were identified and also compared it to what I was seeing in order to see if it was accurate. Since the app was able to catch any objects that could serve as a hazard, I only recorded the types of objects in the table below.

\begin{tabular}{|l|l|}
\hline Type of Area & Types of Object \\
\hline Busy Street & Cars, traffic lights, curbs, fire hydrants \\
\hline Shopping Area & Potted plant, people, lamppost, cars \\
\hline Pedestrian Area & People, trash cars, statues \\
\hline
\end{tabular}

In the table above, it is evident that many different types of objects were identified in each type of area, demonstrating this app's capabilities.

For my second experiment, I assessed different indoor situations, including different rooms and different shops. Since I had to test out situations both inside the house and indoor moments outside the house, there were more independent variables to test out. I ended up having a total of 5 independent variables: bedroom, bathroom, living room, grocery store, and mall. My dependent variables were the same as Experiment 1 - I was observing the objects that the app announced. The app was very specific in what type of object was announced, allowing me to see that it would be very helpful to the visually impaired.

\begin{tabular}{|l|l|}
\hline Type of Area & Types of Object \\
\hline Bedroom & Bed, desk, chair, lamp, picture frame \\
\hline Bathroom & Sink, toilet, bathtub, soap dish \\
\hline Living Room & Couch, table, chairs, lamp, plates \\
\hline Grocery Store & Various produce, tables, counters, baskets, people \\
\hline Mall & Trash can, people, tables, stands \\
\hline
\end{tabular}

Similar to Experiment 1, the table illustrating Experiment 2's results offer a variety of objects identified by the app.

Before conducting the experiment, I had several versions of the app that were not as adequate, so I had to make several changes to ensure that the information from the above experiments would be utilized best. 
Overall, the results of my experiments illustrate that this app is very sufficient. Upon scanning the camera, the app caught any object that could possibly be a hazard for the visually impaired, showing that it has met my expectation. It is essential that this app works for any environment, and the results demonstrate that this app will be useful both inside and outside the house.

For example, near the shopping plaza, the app was even able to identify a large potted plant, which is very specific and important, because it could be a falling hazard. Additionally, in the house, there are a lot of objects that are easily shattered, so this app being able to identify them offers a greater capability than a cane.

These results show that this app will truly allow the visually impaired to explore and have more freedom than before.

\section{RELATED WORK}

This academic journal analyzes how location navigation is very simple for the outdoors, but is very lacking in indoor environments. So, the article proposes that buildings set up radio frequency identification (RFID) tags [14] in order to provide the current location of a visually impaired person to help them navigate indoors [1]. This work emphasizes the involvement of building managers or agents, since this is a whole system that must be installed. In contrast, the app I have created is more tailored to individual people and their desire to explore. While RFID tags help a visually impaired person know their location indoors on the way to their destination, the "Sonar Object Detector" App is purely for detecting potential hazards on a visually impaired person's path. Additionally, the app works both indoors and outdoors, while the article is proposing just an indoor system.

L. Ran, S. Helal, and S. Moore recognizes that there is a lack of navigation system for the visually impaired that works both outdoors and indoors $[2,15]$. So, they propose a system called Drishti, a wearable computer with wireless connection and vocal communication, to help direct a visually impaired person wherever they go, both indoors and outdoors. The proposed system of Drishti is very similar to the "Sonar Object Detector" App in the way that it works both indoors and outdoors. The app I have created is just a smartphone app in comparison to Drishti being a wearable computer. As a result, Drishti is more likely to be more like a person offering aid to a visually impaired person than an app announcing objects. However, Drishti is most likely more expensive than the app, which is free.

This research paper, published in 1996, describes an experiment they conducted on how GPS systems can work with the visually impaired [3]. The successful experiment tested how GPS receivers on a wireless phone carried by a visually impaired person would identify their location relative to their environment. This paper is rather similar to my own, in the way that a GPS is involved, especially when synchronizing with Google Maps. It is notable that this paper was published in 1996, so phones have made great improvements since then. The "Sonar Object Detector" App involves using a smartphone app, which did not exist back then. So, this paper is similar to mine, only a bit more outdated.

\section{CONCLUSion AND Future WORK}

In this project, I proposed using a smartphone app to scan visually impaired person's surroundings in order to point out any potential hazards. I was successful in creating an app that would accurately identify any hazard in most types of situations a visually impaired person would encounter. 
In order to test how accurate my app was after I made a seemingly successful beta version, I had to conduct an experiment. In the first experiment, I tested what kind of objects the app would identify in 3 outdoor environments - a busy street, a shopping plaza, and a pedestrian-filled area. The "Sonar Object Detector" App identified a variety of objects, ranging from a fire hydrant to a truck to a potted plant. In my second and final experiment, I tested how the app would work in 5 indoor environments - a bedroom, a bathroom, a living room, a grocery store, and a mall. I chose these locations thinking about locations a visually impaired person would want to travel to/through independently. Just like with Experiment 1, the "Sonar Object Detector" app was successful in announcing objects ranging from plates to a lamp to baskets to furniture. Overall, the experiment showed that the app was very capable and adaptable in most situations.

During my creation of the app, I encountered three main challenges. First, I had little to none coding experience so I struggled with creating an app from scratch. So, I reached out to a professor who helped guide me through the process. Second, I was creating the app from an Android perspective and have encountered several obstacles on adapting it to iOS, so I have yet to overcome this challenge. Third, I struggled on how to spread knowledge of this app to the visually impaired. As a solution, I reached out to the American Foundation for the Blind who have partnerships for those wanting to help. Through them, I was able to spread the knowledge of this app much farther.

Like stated above, the biggest limitation is that this app is only available on Android. This is significant because iOS devices are so commonly used, meaning that we are currently leaving out a large sector of the visually impaired. So, it is imperative that more work on the app must occur. Furthermore, the accuracy of this app could use more improvement. As of now, the "Sonar Object Detection" App can accurately identify any possible hazards, but only at a short distance. That is sufficient in making sure a visually impaired person is safe, but if the app was able to announce objects that are at a greater distance (and announce the distance as well), this would allow a visually impaired person much more knowledge of their surroundings.

This app is currently available for download on Androids, and I believe that I should not wait until the iOS version is available so people can access it as soon as possible. However, I have already begun working on the iOS version and have made great strides and plan to have that out once I have tested its accuracy. Additionally, I plan to adapt what this app offers to really allow a visually impaired person to navigate the world freely.

\section{REFERENCES}

[1] S. Chumkamon, P. Tuvaphanthaphiphat and P. Keeratiwintakorn, "A Blind Navigation System Using RFID for Indoor Environments," 2008 5th International Conference on Electrical Engineering/Electronics, Computer, Telecommunications and Information Technology, Krabi, Thailand, 2008, pp. 765-768, doi: 10.1109/ECTICON.2008.4600543.

[2] L. Ran, S. Helal and S. Moore, "Drishti: an integrated indoor/outdoor blind navigation system and service," Second IEEE Annual Conference on Pervasive Computing and Communications, 2004. Proceedings of the, Orlando, FL, USA, 2004, pp. 23-30, doi: 10.1109/PERCOM.2004.1276842.

[3] H. Makino, I. Ishii and M. Nakashizuka, "Development of navigation system for the blind using GPS and mobile phone combination," Proceedings of 18th Annual International Conference of the IEEE Engineering in Medicine and Biology Society, Amsterdam, Netherlands, 1996, pp. 506-507 vol.2, doi: 10.1109/IEMBS.1996.651838.

[4] Svennerberg, Gabriel. Beginning Google Maps API 3. Apress, 2010.

[5] Maps, Goolge. "Google maps." Dipetik Desember 14 (2015): 2015.

[6] Furlan, Cornelio A., and Vivian Su. "Sonar Walking Stick." (2014). 
[7] Developers, Android. "What is android." Dosegljivo: http://www. academia. edu/download/30551848/andoid--tech. pdf (2011).

[8] Gargenta, Marko. Learning android. " O'Reilly Media, Inc.", 2011.

[9] Alliance, Open Handset. "Android overview." Open Handset Alliance 8 (2011): 88-91.

[10] Ahmad, Mohd Shahdi, Nur Emyra Musa, Rathidevi Nadarajah, Rosilah Hassan, and Nor Effendy Othman. "Comparison between android and iOS Operating System in terms of security." In 2013 8th International Conference on Information Technology in Asia (CITA), pp. 1-4. IEEE, 2013.

[11] Nolan, Carson Y., and Cleves J. Kederis. "Perceptual Factors in Braille Word Recognition. (American Foundation for the Blind. Research Series No. 20)." (1969).

[12] Josephson, Eric. "The Social Life and Blind People. American Foundation for the Blind Research Series No. 19." (1968).

[13] Harley Jr, Randall K. "Verbalism Among Blind Children; An Investigation and Analysis. American Foundation for the Blind Research Series, Number 10." (1963).

[14] Roberts, Chris M. "Radio frequency identification (RFID)." Computers \& security 25, no. 1 (2006): 18-26.

[15] Helal, Abdelsalam, Steven Edwin Moore, and Balaji Ramachandran. "Drishti: An integrated navigation system for visually impaired and disabled." In Proceedings fifth international symposium on wearable computers, pp. 149-156. IEEE, 2001.

(C) 2021 By AIRCC Publishing Corporation. This article is published under the Creative Commons Attribution (CC BY) license. 